\title{
EFFETS DE L'EXPOSITION À UN INSECTICIDE CARBAMATE, LE CARBOFURAN, SUR LES PERFORMANCES DE JEUNES STADES DE BROCHET (ESOX LUCIUS L., 1758) : RÉSULTATS PRÉLIMINAIRES.
}

\author{
G. AMBLARD (1), C. BRY (1), J.P. TOUTANT (2) et M. ARPAGAUS (2)
}

(1) INRA, Unité d'Ecotoxicologie Aquatique, 65 rue de Saint-Brieuc, 35042 Rennes Cedex, France.

(2) INRA, Laboratoire de Différenciation Cellulaire et Croissance, 2 Place Pierre Viala, 34060 Montpellier Cedex 1, France.

\section{RÉSUMÉ}

Des lots de jeunes brochets au stade embryon libre, stabulés dans des béchers en verre, ont été exposés en conditions statiques à des solutions $\left(0-250 \mu \mathrm{g} . \mathrm{L}^{-1}\right)$ de carbofuran, un insecticide carbamate inhibiteur de l'activité de l'acétylcholinestérase (AchE). A partir de $10 \mu \mathrm{g} . \mathrm{L}^{-1}$, une inhibition significative de l'activité globale de l'AchE des embryons libres a été constatée. A partir de $50 \mu \mathrm{g} . \mathrm{L}^{-1}$, une diminution significative de la hauteur moyenne de fixation des embryons libres sur les parois des béchers a été observée. L'exposition au carbofuran n'a pas eu d'effet significatif sur le nombre de proies zooplanctoniques ingérées par les jeunes brochets parvenus au début de la phase larvaire ; une diminution de ce paramètre en fonction de concentrations croissantes de carbofuran a cependant été enregistrée. A $250 \mu \mathrm{g} . \mathrm{L}^{-1}$, la résorption de la vésicule vitelline ne s'effectuant plus que très partiellement, les embryons libres exposés ne se sont pas transformés en larves.

Cette étude montre que l'exposition à des concentrations sublétales de carbofuran est susceptible d'affecter le développement ontogénétique et les performances de jeunes stades de brochet. Un approfondissement de ces travaux est envisagé dans différentes directions.

Mots-clés : insecticide, brochet, embryon libre, larve, réponses comportementales.

\author{
EFFECTS OF CARBOFURAN, A CARBAMATE INSECTICIDE, ON \\ BIOLOGICAL PERFORMANCES OF YOUNG PIKE (ESOX LUCIUS L., 1758) : \\ PRELIMINARY RESULTS.
}

\section{ABSTRACT}

Pike free embryos were introduced into glass beakers and exposed under static conditions to solutions $\left(0-250 \mu \mathrm{g} \cdot \mathrm{L}^{-1}\right)$ of carbofuran, a carbamate insecticide inhibiting acetylcholinesterase (AchE) activity. From a carbofuran concentration of $10 \mu \mathrm{g.L} \mathrm{L}^{-1}$, the overall AchE activity in free embryos was significantly inhibited. From $50 \mu \mathrm{g} . \mathrm{L}^{-1}$, the mean 
level of attachment of free embryos to the beakers walls decreased significantly. The number of zooplanktonic prey ingested by pike larvae was not significantly affected by exposure to carbofuran ; however, this parameter tended to decrease with increasing concentrations of carbofuran. In free embryos exposed to $250 \mu \mathrm{g} . \mathrm{L}$ ' carbofuran, yolk sac resorption was very incomplete and the transformation into larvae did not take place.

The present study demonstrates that sublethal exposures to carbofuran may affect ontogenetic changes and biological performances in pike young stages. Further developments of this work are planned in several directions.

Key-words : insecticide, pike, free embryo, larva, behavioural responses.

\section{INTRODUCTION}

Le carbofuran est un insecticide à large spectre utilisé pour le traitement des semences dans le cadre de différentes cultures: cultures légumières, maïs, soja, tournesol, arbres, arbustes d'ornement, cultures florales, fraisier (ACTA, 1996). Cette molécule appartient à la famille des carbamates et agit par inhibition de l'activité des cholinestérases (MINEAU, 1991). Ce mode d'action peu spécifique a pour conséquence la possibilité d'effets toxiques chez un grand nombre d'organismes non-cibles, dont des organismes aquatiques puisque ce composé, mobile et relativement soluble dans l'eau, est susceptible de migrer rapidement vers les eaux superficielles après application (GUPTA, 1994). Ainsi des effets sur certaines catégories de macroinvertébrés aquatiques ont-ils été mis en évidence, au niveau comportemental (GRANT et al., 1983 ; PAWAR et KATDARE, 1984a) et au niveau populationnel (WAYLAND et BOAG, 1990). Chez les poissons, les effets sublétaux observés sur le plan biochimique concernent des modifications des teneurs cérébrales en acétylcholinestérase (JASH et BHATTACHARYA, 1983) et en plusieurs neurotransmetteurs (GOPAL et RAM, 1995), ainsi que des niveaux des transaminases dans la circulation et des phosphatases dans différents organes (VERMA et al., 1981a et b). Sur le plan comportemental, des anomalies incluant l'hypoactivité, la perte d'équilibre, la déformation de l'axe vertébral, la paralysie de la région buccale, des opercules ou encore de l'ensemble du corps, ont été rapportées (TROTTER et al., 1991). Chez le carassin exposé au carbofuran, des modifications de l'activité de nage, des relations sociales et de l'orientation par rapport à un filtrat alimentaire, ont été décrites récemment (SAGLIO et al., 1996). Les effets comportementaux sont cependant assez peu étudiés en toxicologie chez le poisson, et en particulier chez les jeunes stades, alors qu'ils présentent l'intérêt de constituer des réponses intégrées au niveau de l'organisme. Par ailleurs, les jeunes stades de poissons d'eau calme (cyprinidés, ésocidés) figurent parmi les organismes potentiellement vulnérables, puisque les périodes de début du cycle vital de ces poissons, du début du printemps au début de l'été, recoupent largement le calendrier des traitements au carbofuran (ACTA, 1996). Dans cet esprit, le présent travail a été consacré à une étude expérimentale des effets du carbofuran sur les jeunes stades du brochet (ésocidé). Ces effets ont été étudiés au niveau d'un paramètre biochimique (l'activité de l'acétylcholinestérase totale) et de deux paramètres comportementaux (positionnement et prise alimentaire).

\section{MATÉRIEL ET MÉTHODES}

Des embryons libres de brochet (Esox lucius L., 1758) ont été isolés peu de temps après l'éclosion et répartis dans des béchers de $2 \mathrm{~L}$ en verre, à raison de 20 embryons par bécher. Six lots expérimentaux ont été constitués, en fonction des caractéristiques de l'eau introduite dans les béchers : témoin eau, témoin acétone (solvant de dissolution du 
carbofuran) et quatre lots d'exposition au carbofuran (concentrations testées : 2, 10, 50 et $\left.250 \mu \mathrm{g} . \mathrm{L}^{-1}\right)$. L'expérimentation a été conduite en triplicat ( 3 béchers par lot). Les embryons libres de brochet ayant pour caractéristique d'adhérer de façon intermittente à la végétation ou à une paroi verticale (voir revue par BRY, 1996), le premier paramètre mesuré a été la Hauteur Moyenne de Fixation (HMF) pendant trois jours d'exposition. Après renouvellement des solutions, l'exposition a été poursuivie pendant quatre jours supplémentaires. En fin de vie embryonnaire, cinq embryons libres par bécher ont été congelés en vue de la mesure de l'activité de l'AchE sur individus entiers (ELLMAN et al., 1961). Les autres jeunes brochets sont entrés en phase d'alimentation exogène et le nombre de proies zooplanctoniques ingérées par chaque larve de brochet au cours d'un contact de $4 \mathrm{~h}$ avec du zooplancton calibré a été déterminé par examen des contenus stomacaux. L'analyse statistique des résultats a fait intervenir les tests non paramétriques de Kruskall-Wallis et de Mann-Whitney (SOKAL et ROHLF, 1981).

\section{RÉSULTATS}

La Figure 1 représente les résultats relatifs à la hauteur moyenne de fixation et à l'activité de l'AchE observées dans les différents lots d'embryons libres de brochet. Les

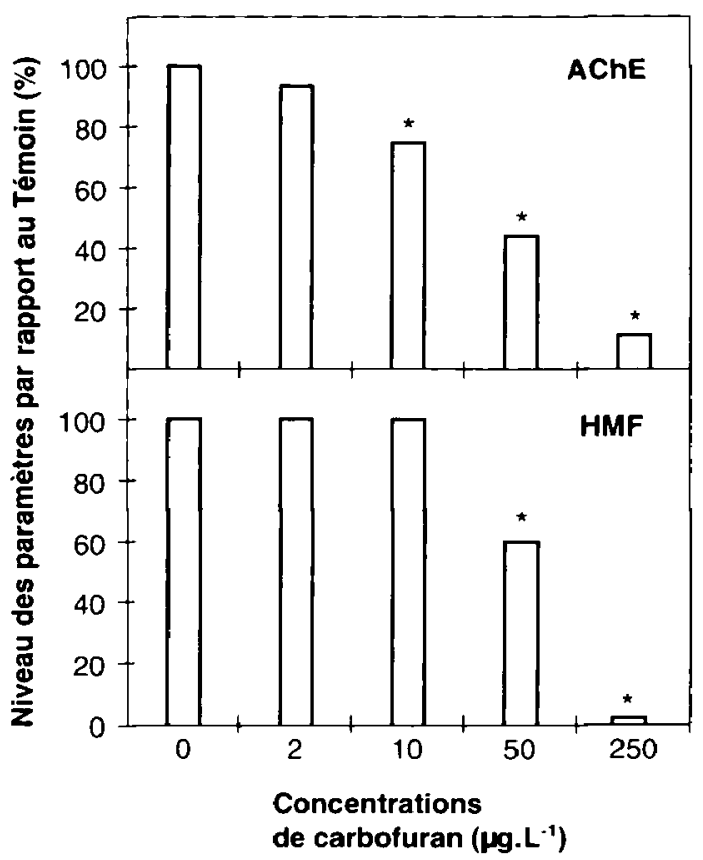

Figure 1

Effets sublétaux du carbofuran (insecticide carbamate) sur des embryons libres de brochet : niveaux relatifs d'un paramètre comportemental (Hauteur Moyenne de Fixation, HMF) et d'un paramètre biochimique (activité enzymatique de l'acétylcholinestérase, AchE) par rapport au témoin (\%), en fonction des concentrations d'exposition au carbofuran.

* Niveau relatif significativement inférieur à $100 \%(p<0,05)$.

\section{Figure 1}

Sublethal effects of carbofuran, a carbamate insecticide, on pike tree embryos : levels of a behavioural parameter (mean height of attachment, HMF) and of a biochemical parameter (acetylcholinesterase enzymatic activity, AchE), expressed in p. cent of control levels and related to carbofuran concentrations.

* Relative level significantly lower than $100 \%(p<0.05)$. 
valeurs de ces deux paramètres n'étant pas significativement différentes chez les "témoins eau " et chez les " témoins acétone ", les résultats ont été exprimés par rapport aux " témoins eau ". A partir d'une concentration de carbofuran de $10 \mu \mathrm{g} . \mathrm{L}$ ", l'activité de l'AchE est inhibée de façon significative $(p<0,05)$; à 50 et $250 \mu \mathrm{g}$.L', les activités résiduelles exprimées en pourcentage du niveau du " témoin eau "s'élèvent respectivement à 43,9 et $11,2 \%$. A $50 \mu \mathrm{g}$. L' de carbofuran, la hauteur moyenne de fixation des embryons libres chute à $64 \%$ du niveau des témoins $(p<0,05)$ et devient nulle à $250 \mu \mathrm{g} . \mathrm{L}^{\cdot}$. Le nombre moyen de proies zooplanctoniques ingérées diminue dès $2 \mu \mathrm{g} \cdot \mathrm{L}^{-1}(84 \%$ du niveau des témoins). Cette diminution se poursuit pour des concentrations de carbofuran de 10 et $50 \mu \mathrm{g}$ L. $^{-1}$ ( 81 et $54 \%$, respectivement) ; elle n'est cependant pas significative au seuil $5 \%$. Les embryons libres exposés à une concentration de carbofuran de $250 \mu \mathrm{g} . \mathrm{L}^{-1}$ présentent une déformation de l'axe vertébral, ainsi qu'une quasi-absence de résorption de la vésicule vitelline; corrélativement, leur longueur moyenne $(9,6 \mathrm{~mm})$ est significativement inférieure à celle des témoins $(12,6 \mathrm{~mm} ; \mathrm{p}<0,001)$ et ces jeunes poissons, dont le stade ontogénétique est plus proche de celui d'embryon libre récemment éclos que de celui de larve (stade atteint par les témoins), ne possèdent aucune capacité d'alimentation exogène.

\section{DISCUSSION - CONCLUSION}

La présente étude, effectuée en parallèle sur un plan biochimique et sur un plan comportemental, démontre la sensibilité d'embryons libres de brochet vis-à-vis de concentrations sublétales de carbofuran. L'effet biochimique, visible à partir de $10 \mu \mathrm{g} . \mathrm{L}^{-1}$, devance les effets observés à des niveaux biologiques plus " intégrés " qui, compte tenu de la gamme restreinte des concentrations expérimentées, ne sont détectables qu'à partir de $50 \mu \mathrm{g} . \mathrm{L}^{-1}$. Ce résultat est à rapprocher de ceux de HEATH et al. (1993) : ces auteurs ont en effet observé une diminution de l'activité spontanée chez des larves de bar rayé exposées à $79 \mu \mathrm{g} . \mathrm{L}^{-1}$ de carbofuran pendant 4 jours. A $50 \mu \mathrm{g} . \mathrm{L}^{-1}$, le taux d'inhibition de l'activité de l'AchE est proche de $50 \%$. Un taux d'inhibition de $50 \%$ a été observé chez le poisson-chat Ictalurus punctatus au niveau cérébral, pour une concentration d'exposition nettement supérieure (190 $\mu \mathrm{g} . \mathrm{L}^{-1}$; NRCC, 1979). L'absence d'effets comportementaux chez l'embryon libre de brochet après une exposition à $10 \mu \mathrm{g} \cdot \mathrm{L}^{-1}$ de carbofuran est à rapprocher des concentrations maximales acceptables pour le cyprinidé Cyprinodon variegatus, déterminées par HANSEN et PARRISH après exposition chronique (périodes de 19 semaines) de différents stades du cycle vital de cette espèce (In TROTTER et al., 1991) : 15-23 $\mu \mathrm{g} . \mathrm{L}^{-1}$. Par ailleurs, des effets toxiques et tératogéniques (courbure de l'axe du corps, retards de croissance et de pigmentation, anomalies comportementales) ont été également observés chez des embryons et des têtards de grenouille (Microhyla ornata) exposés au carbofuran (PAWAR et KATDARE, 1984b). Nous avons observé une diminution de la prise alimentaire chez de jeunes larves de brochet préalablement exposées au carbofuran : à $50 \mu \mathrm{g} \cdot \mathrm{L}^{-1}$, cette diminution - d'un facteur 2 environ - est du même ordre que celles obtenues pour les deux autres paramètres étudiés. Elle n'est cependant pas significative au seuil $5 \%$, en raison d'une forte variabilité interindividuelle du paramètre " nombre de proies ingérées par unité de temps".

If est clair, d'après les données de la littérature et celles de notre étude, que le carbofuran présente un degré élevé de neurotoxicité à l'égard des poissons et de nombreux invertébrés aquatiques (GUPTA, 1994). Cependant, dans le contexte particulier de la contamination des eaux superficielles par des pesticides en Bretagne, le risque d'observer chez le brochet les altérations décrites ci-dessus paraît faible, compte tenu du niveau modéré des concentrations maximales enregistrées $\left(0,2-0,3 \mu \mathrm{g} . \mathrm{L}^{\cdot \cdot}\right.$; GUIHO, 1995) et du caractère fugace de la présence du carbofuran dans ces eaux. L'éventualité d'une accumulation de carbofuran dans certains tissus des jeunes stades de brochet et d'une toxicité à des stades ultérieurs du cycle vital est peu vraisemblable, plusieurs études 
ayant montré l'absence d'une bioconcentration significative de ce composé chez les poissons et l'élimination rapide des métabolites du carbofuran par les poissons, après arrêt de l'exposition (revue par TROTTER et al., 1991).

Un approfondissement de la présente étude est envisagé dans plusieurs directions :

(1) confirmer les effets sublétaux du carbofuran chez les jeunes stades de brochet en utilisant une gamme de concentrations plus dense, en particulier dans l'intervalle 10-100 $\mu \mathrm{g} . \mathrm{L}^{\cdot 1}$;

(2) l'AchE existant sous différentes formes moléculaires (TOUTANT et MASSOULIE, 1987) et des néosynthèses de certaines formes ayant été mises en évidence chez le rat après exposition de cultures de myotubes à un agent organophosphoré (BROCKMAN et al., 1986), identifier les formes présentes en fonction du contexte de contamination ;

(3) dans une perspective de biologie comparée, examiner les effets sublétaux du carbofuran chez un poisson cyprinidé d'eau calme, en travaillant sur les mêmes stades et les mêmes paramètres que dans le cas du brochet.

\section{BIBLIOGRAPHIE}

ACTA, 1996. Index phytosanitaire. Association de Coordination Technique Agricole, Paris.

BROCKMAN S.K., USIAK M.F., YOUNKIN S.G., 1986. Assembly of monomeric acetylcholinesterase into tetrametric and asymmetric forms. Journal of Biological Chemistry, 261, 1201-1207.

BRY C., 1996. Role of vegetation in the life cycle of pike. In CRAIG J.F. (ed.), Pike : Biology and exploitation, Chapman and Hall, London, 45-67.

ELLMAN G.L., COURTNEY K.O., ANDRES V., FURTHERSTONE R.M., 1961. A new and rapid colorimetric determination of acetylcholinesterase activity. Biochemical Pharmacology, 7, 88-89.

GOPAL K., RAM M., 1995. Alteration in the neurotransmitter levels in the brain of the freshwater snakehead fish (Channa punctatus) exposed to carbofuran. Ecotoxicology, 4, 1-4.

GRANT I.F., EGAN E.A., ALEXANDER M., 1983. Pesticides to control ostracods grazing on blue-green algae. Soil Biol. Biochem., 15, 193.

GUIHO M., 1995. Les réseaux de surveillance : Résultats, évolution. In Actes du colloque CORPEP / Bretagne Eau Pure Qualité des eaux et produits phytosanitaires : Du diagnostic à l'action, Rennes, 27 novembre 1995, 19-30.

GUPTA R.C., 1994. Carbofuran toxicity. J. Toxicol. Environ. Health, 43, 383-418.

HEATH A.G., CECH J.J., ZINKL J.G., FINLAYSON B., FUJIMURA R., 1993. Sublethal effects of methyl parathion, carbofuran, and molinate on larval striped bass. American Fisheries Society Symposium, 14, 17-28.

JASH N.B., BHATTACHARYA S., 1983. Delayed toxicity of carbofuran in freshwater teleost Channa punctatus. Indian J. Exp. Biol., 17, 693-697.

MINEAU P., 1991. Cholinesterase-inhibiting Insecticides. Elsevier Science Publishers, Amsterdam.

NRCC, 1979. Carbofuran : Criteria for Interpreting the Effects of Its Use on Environmental Quality. NRCC N $\mathrm{N}^{\circ} 16740$, National Research Council of Canada, Ottawa.

PAWAR K.R., KATDARE M., 1984a. Effect of sublethal and lethal concentrations of fenitrothion, BHC and carbofuran on behaviour and oxygen consumption on the freshwater prawn Macrobrachium kistnensis (Tiwari). Arch. Hydrobiol., 99, 398. 
PAWAR K.R., KATDARE M., 1984b. Toxic and teratogenic effects of fenitrothion, BHC and carbofuran on embryonic development of the frog Microhyla ornata. Toxicol. Lett., 22, 7-13.

SAGLIO P., TRIJASSE S., AZAM D., 1996. Behavioral effects of waterborne carbofuran in goldfish. Arch. Environ. Contam. Toxicol., 31, 232-238.

SOKAL R.R., ROHLF F.J., 1981. Biometry. Freeman, New York.

TOUTANT J.P., MASSOULIE J., 1987. Acetylcholinesterase. In KENNY et TURNER (eds), Mammalian ectoenzymes, chapitre 11, 289-328, Elsevier Science Publishers, Amsterdam.

TROTTER D.M., KENT R.A., WONG M.P., 1991. Aquatic Fate and Effect of Carbofuran. Critical Reviews in Environmental Control, 21(2), 137-176.

VERMA S.R., RANI S., DALELA R.C., 1981a. Isolated and combined effects of pesticides on serum transaminases in Mystus vittatus (African catfish). Toxicol. Lett., 8, 67.

VERMA S.R., RANI S., DALELA R.C., 1981b. Pesticide-induced physiological alterations in certain tissues of a fish, Mystus vittatus (African catfish). Toxicol. Lett., 9, 327.

WAYLAND M., BOAG D.A., 1990. Toxicity of carbofuran to selected macroinvertebrates in prairie ponds. Bull. Environ. Contam. Toxicol., 45, 74. 